\title{
Phase Structure and Heat Generation in the Co-Precipitated Magnetite Nanoparticles
}

\author{
P. Pawlik, M. Pruba, K. Pawlik and K. Kotynia \\ Institute of Physics, Częstochowa University of Technology, al. Armii Krajowej 19, 42-200 Częstochowa, Poland
}

\begin{abstract}
In the present studies the phase structure and magnetic ordering of magnetite nanoparticles were investigated. The powder samples were obtained by co-precipitation from $\mathrm{Fe}(\mathrm{III})$ and $\mathrm{Fe}(\mathrm{II})$ salts. $\mathrm{SiO}_{2}$ coating was performed using the modified Stöber process. X-ray diffraction studies revealed presence of the only one $\mathrm{Fe}_{3} \mathrm{O}_{4}$ crystalline phase both for the non-coated and $\mathrm{SiO}_{2}$-coated samples. The Mössbauer studies have shown differences in shapes of measured spectral lines for $\mathrm{Fe}_{3} \mathrm{O}_{4}$ particles subjected to the Stöber process and non-coated particles. The heating efficiency was measured for glycerol dispersed nanoparticles. The analysis have shown changes in specific loss power depending on the concentration of the nanoparticles and amplitude of alternating magnetic field.
\end{abstract}

DOI: 10.12693/APhysPolA.131.1217

PACS/topics: 75.47.Lx, 78.67.Bf, 78.70.Ck, 76.80.+y, 87.50.S-

\section{Introduction}

Almost all cells in the human body may degenerate and create a cancer. The tumor consist of a number of cells that grow in an unrestrained way, thus they become deformed and their metabolism is different than in normal tissue. The cause of degradation of genetic material can originate in a variety of factors such as carcinogens, or viruses [1]. Up to now it is impossible to consider modern cancer treatments without adjuvant therapies. The mechanism of those cancer treatments mainly consists of stimulation of the immune system in order to inhibit growth of deformed cells and improve the immunity. One of such adjuvant therapies is the hyperthermia [2]. It is a sort of tumor treatment, in which body tissue is exposed to the elevated temperature. In general, the cancer cells are more sensitive to the high temperature than the healthy ones. There are few variants of hyperthermia which can be divided into the active and passive treatments [3]. The active hyperthermia is focused on application of chemical substances in order to induce a fever, while the passive hyperthermia consists of using the physical methods to deliver a heat into the body. The magnetic variant of hyperthermia deals with the ability of the magnetic nanoparticles to be heated when the RF alternating magnetic field is applied. However, there are safety limits concerning both applied magnetic field and frequency range. So that amplitude of the magnetic field $H$ should not be higher than $15 \mathrm{kA} / \mathrm{m}$, while the frequency has to be in the range $0.05 \mathrm{MHz}<f<1.5 \mathrm{MHz}[4]$.

Numerous reports on ferromagnetic elemental and intermetallic nanoparticles used for the hyperthermia studies or other medical applications were published until now [5-7]. Interesting investigations were presented in [5] for composites containing Ni nanocrystallites entrapped in a carbon matrix, produced by frontal polymerization of a $\mathrm{Ni}$ acrylamide nitrate complex, followed by its pyrolysis. For this material the particle size was controlled by the temperature of pyrolysis, thus leading to the super- paramagnetic or ferromagnetic ordering of Ni nanoparticles. For the superparamagnetic Ni nanoparticles a heating mechanism was proven to be associated with the Néel relaxation. However, it was shown that ferromagnetic ordering leads to the most effective heating process. This is a similar result to those obtained for iron-oxide aggregates of nanoparticles $[8,9]$. It was shown that the superparamagnetic iron-oxide (SPM) particles were aggregated due to interparticle magnetic interaction that led to an effective magnetic moment and ferromagneticlike behavior with non-zero hysteresis. This resulted in generation of substantial heat when exposed to AMF [8].

A commonly accepted magnetic solute used as a drug delivery carrier or in magnetic particle imaging (MPI) by the relevant medical institutions in many countries are magnetite $\left(\mathrm{FeFe}_{2} \mathrm{O}_{4}\right)$ nanoparticles. Therefore this material is considered as a good candidate for application in the hyperthermia treatment.

In the present work the phase structure and magnetic ordering of the iron-oxide nanoparticles obtained by coprecipitation method were studied. Furthermore, based on behavior of glycerol solution of iron-oxide nanoparticles in RF magnetic field, the possibility of their use for hyperthermia treatment has been assessed.

\section{Samples preparation and experimental method}

The iron-oxide nanoparticles were produced by the coprecipitation method, using the solution of $\mathrm{FeCl}_{3} \cdot 6 \mathrm{H}_{2} \mathrm{O}$ and $\mathrm{FeSO}_{4} \cdot 7 \mathrm{H}_{2} \mathrm{O}$ salts as precursors of $\mathrm{Fe}^{3+}$ and $\mathrm{Fe}^{2+}$ ions with their molar ratio $\mathrm{Fe}^{2+}: \mathrm{Fe}^{3+}=0.67$. The process was carried out under the $\mathrm{N}_{2}$ atmosphere at $60^{\circ} \mathrm{C}$. Ammonium hydroxide solution was added dropwise under vigorous stirring. The $\mathrm{pH}$ during process has changed from 12 to $\approx 8$. The iron-oxide powder was separated by the permanent magnet and washed several times in distilled water to reach natural $\mathrm{pH}$. Subsequently the powder was air dried for $24 \mathrm{~h}$ at $50^{\circ} \mathrm{C}$. Core-shell structure was obtained using the modified Stöber process $[10,11]$. 
In the reaction $30 \mathrm{mg}$ of iron-oxide nanoparticles were dispersed in $100 \mathrm{ml}$ of 2-propanol, $12 \mathrm{ml}$ of distilled water and $15 \mathrm{ml}$ of $25 \%$ ammonia solution and sonicated for $1 \mathrm{~h}$. Subsequently, $3.2 \mathrm{ml}$ of tetraethyl orthosilicate (TEOS) was added and sonicated for another $6 \mathrm{~h}$. The powder was filtered, washed with 2-propanol and dried at $20^{\circ} \mathrm{C}$ for $24 \mathrm{~h}$. The phase structure of the powder was determined using Bruker D8 Advance X-ray diffractometer in the Bragg-Brentano configuration. Measurements were performed using $\mathrm{Cu} K_{\alpha}$ radiation (linear focus $24 \mathrm{~mm}$ and primary divergence slit $0.6 \mathrm{~mm}$ ) and LynxEye detector (Ni filter of $K_{\beta}$ was located on detector side) in the range of $2 \Theta$ from 20 to $100 \mathrm{deg}$ in the steps of $0.02 \mathrm{deg}$. The XRD patterns were analysed using DIFFRAC.SUITE TOPAS 4.2 software. NIST LaB 6 standard sample was measured to calculate the emission profile using the Thompson-Cox-Hastings pseudo-Voigt (TCHZ) peak type function. The Mössbauer studies were carried out in transmission mode using ${ }^{57} \mathrm{Co}$ source in $\mathrm{Rh}$ matrix of the activity $50 \mathrm{mCi}$. The analysis of spectra was performed using Normos software. For the time dependence of temperature characteristics the magnetite nanoparticles were dispersed in glycerol and sonicated for $5 \min [12,13]$.

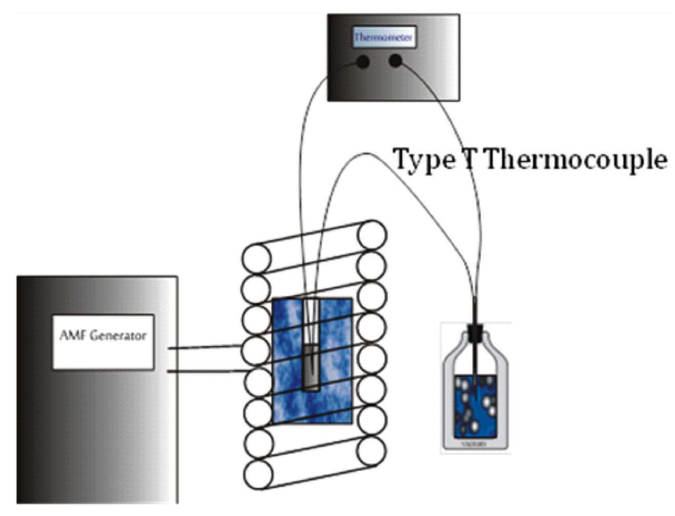

Fig. 1. Schematic diagram of the setup for measurement of the heating efficiency of the magnetite nanoparticles dispersion.

In order to assess the heating efficiency of the magnetite nanoparticles dispersion and specific loss power (SLP), the self-constructed setup was used. The schematic diagram of the design was presented in Fig. 1. The measured sample is placed in the thermally isolated container at the center of the water cooled induction coil. The alternating magnetic field generator (AMF) was working at constant frequency of $100 \mathrm{kHz}$. The magnetic field was measured indirectly by the Rogowski coil while the temperature using $T$-type micro-thermocouple.

\section{Results and discussion}

In Fig. 2 the X-ray diffraction patterns measured for synthesized powders were presented. The phase analysis has proven a presence of the only one $\mathrm{Fe}_{3} \mathrm{O}_{4}$ crystalline phase (of the inverse spinel type; the space group: $F d$ $3 m$ ) for both non-coated and silica-coated samples. In order to determine the unit cell parameters and crystallite sizes, the Rietveld refinement has been used. Taking into account the instrument contribution to the diffraction line broadening, the external standard method for calibration of the X-ray diffractometer with NIST LaB 6 of 660 series sample was used.

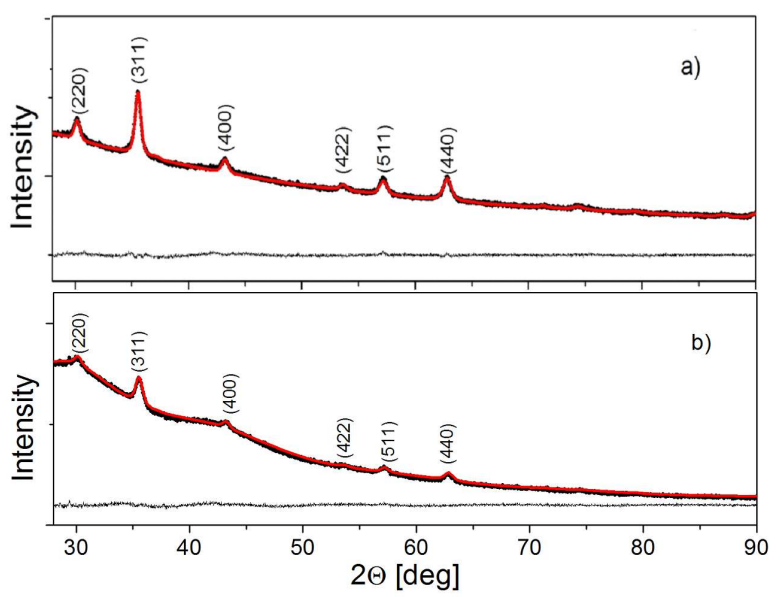

Fig. 2. The measured (black line) and calculated (red line) XRD patterns for the non-coated (a) and $\mathrm{SiO}_{2}$ coated (b) nanoparticles of the $\mathrm{Fe}_{3} \mathrm{O}_{4}$ obtained by the co-precipitation process.

Results of the Rietveld refinement performed for the iron-oxide powder samples are collected in Table I. Similar values of the unit cell parameter for both non-coated and $\mathrm{SiO}_{2}$-coated $\mathrm{Fe}_{3} \mathrm{O}_{4}$ particles were estimated. Determined unit cell parameter $a$ of $8.35 \AA$ is larger than initial value used in calculations $(8.32 \AA)$, which might be caused by non-stoichiometric composition of the sample and distribution of sizes of precipitating magnetite nanoparticles. The average crystallite size was determined using whole powder pattern method. The average crystallite size of $10 \mathrm{~nm}$ calculated for non-coated $\mathrm{Fe}_{3} \mathrm{O}_{4}$ particles was slightly lower than this for the $\mathrm{SiO}_{2}$-coated magnetite (of $15 \mathrm{~nm}$ ). This difference for coated nanoparticles might be due to the fluctuation in the lattice spacing by the layers of $\mathrm{SiO}_{2}$ on the surface of $\mathrm{Fe}_{3} \mathrm{O}_{4}$ nanoparticles. For the silica coated nanoparticles the characteristic peak for $\mathrm{SiO}_{2}$ is overlapped by the (200) reflex corresponding to the magnetite. Even though the XRD studies have not revealed clear diffraction peaks coming from silica coating, evident change of the diffraction pattern was shown. This may suggest that the $\mathrm{SiO}_{2}$ coating is not very thick and/or forms amorphous layer [14].

Thus in order to confirm a presence of the $\mathrm{SiO}_{2}$ coating layer on the surface of the magnetite nanoparticles, the additional Mössbauer specra (MS) analysis was performed. Furthermore, MS is a method that allows to determine the magnetic ordering in the studied material as it reflects its hyperfine interatomic interactions. This is 
TABLE I

Refined/calculated parameters Volume weighted coherently diffracting domain sizes $\left(L_{v o l}\right)$; criteria of fit: $R_{\text {exp }}-R$-expected, $R_{w p}-R$-weighted pattern and gof - goodness of fit for Rietveld refinement of XRD patterns.

\begin{tabular}{c|c|c|c|c|c}
\hline \hline & $R_{\text {exp }}$ & $R_{w p}$ & gof & $a[\AA]$ & $L_{\text {vol }}[\mathrm{nm}]$ \\
\hline starting values & & & & 8.32 & \\
$\mathrm{Fe}_{3} \mathrm{O}_{4}$ & 0.789 & 0.862 & 1.092 & $8.360 \pm 0.001$ & 10.8 \\
$\mathrm{Fe}_{3} \mathrm{O}_{4} @ \mathrm{SiO}_{2}$ & 0.859 & 1.041 & 1.212 & $8.354 \pm 0.002$ & 15.8
\end{tabular}

complementary technique to the magnetization vs. temperature measurements in zero-field cooling (ZFC) and field cooling (FC) mode. However, the second technique is not conclusive for the $\mathrm{Fe}_{3} \mathrm{O}_{4}$ nanoparticles, as the increase of magnetization for ZFC curve attributed to the blocking temperature of superparamagnetic particles at low temperatures is concealed by the Verwey transition around $130 \mathrm{~K}$ [15]. This transition involves both crystallographic and electronic rearrangement of the magnetite phase.

The transmission Mössbauer spectra measured for noncoated and silica coated samples are shown in Fig. 3. In the inverse spinel type structure of the magnetite, two crystallographically nonequivalent positions of Fe ions are present. In such structure half of the $\mathrm{Fe}^{3+}$ ions occupy 8 tetrahedral sites while both remaining $\mathrm{Fe}^{3+}$ and all $\mathrm{Fe}^{2+}$ ions occupy 16 octahedral positions. Therefore in the Mössbauer spectra analysis of bulk samples a presence of $\mathrm{Fe}_{3} \mathrm{O}_{4}$ phase is represented by two sextet lines corresponding to octahedral and tetrahedral positions of the Fe ions. In case of investigated particles, taking into account only two component sextets in the spectra analysis did not led to the convergence of the fit.

However, as was shown in $[13,16]$ for the nanocrystalline $\mathrm{Fe}_{3} \mathrm{O}_{4}$ phase large surface area and its distortion as well as deviations from stoichiometry led to the additional sextet lines that have to be incorporated into the analysis. Therefore, to obtain a good fit of the experimental lines, four sextet components were used in the analysis. Broadening of the component lines is related to the thermal agitation. Furthermore, in the analysis of Mössbauer spectrum measured for non-coated specimen it was necessary to add additional doublet line to get best fit. The reason for presence of such additional doublet line is due to distribution of sizes of magnetite nanoparticles precipitating in the co-precipitation process. Taking into account the average crystallite size determined using the Rietveld refinement, there is a significant part of those nanoparticles small enough to exhibit superparamagnetic behavior. Considerable difference between the Mössbauer spectra measured for non-coated and silicacoated samples was encountered. In case of $\mathrm{SiO}_{2}$-coated magnetite nanoparticles, apart from four sextets, additional singlet line was used for the fitting. A presence of this line can be attributed to interactions of surface Fe ions of the magnetite nanoparticles with the surrounding

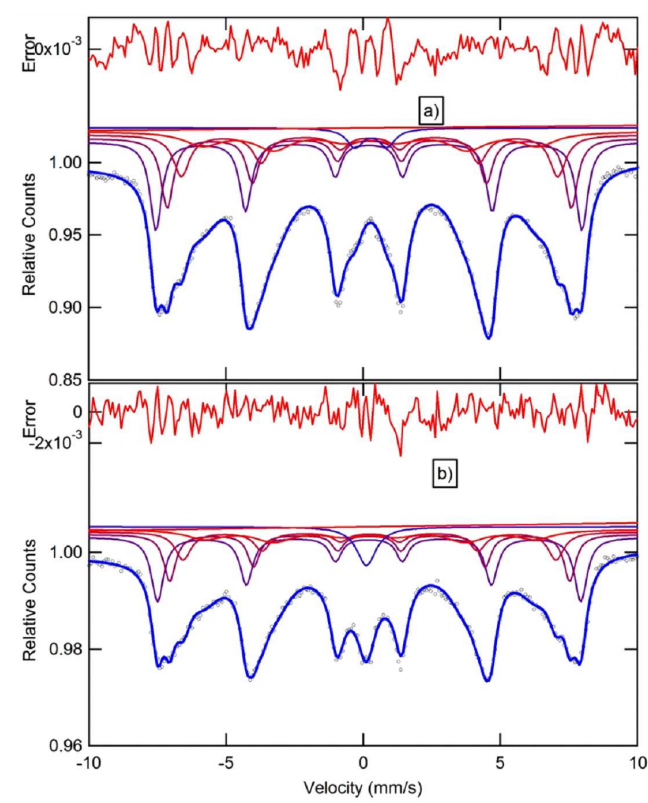

Fig. 3. Room temperature transmission Mössbauer spectra measured for non-coated (a) and silica-coated (b) nanoparticles of magnetite.

silica-coating atoms. Hyperfine fitting parameters calculated for both samples are collected in Table II.

TABLE II

Hyperfine fitting parameters of room temperature transmission Mössbauer spectra measured for magnetite nanoparticles. Hyperfine field $B_{h f}[\mathrm{~T}]$, Isomer shift $I S[\mathrm{~mm} / \mathrm{s}]$, quadrupole splitting $Q S[\mathrm{~mm} / \mathrm{s}]$, and line area $A[\%]$.

\begin{tabular}{c|c|c|c|c|c}
\hline \hline & & $B_{h f}$ & $I S$ & $Q S$ & $A$ \\
\hline & sextet \#1 & 48.12 & 0.3 & 0.001 & 21.3 \\
& sextet \#2 & 45.64 & 0.32 & 0.001 & 16.6 \\
$\mathrm{Fe}_{3} \mathrm{O}_{4}$ & sextet \#3 & 42.51 & 0.33 & 0.001 & 17.0 \\
& sextet \#4 & 37.85 & 0.36 & 0.001 & 42.0 \\
& doublet & - & 0.38 & 1.165 & 4.0 \\
\hline \multirow{5}{*}{$\mathrm{Fe}_{3} \mathrm{O}_{4} @ \mathrm{SiO}_{2}$} & sextet \#1 & 47.87 & 0.31 & 0.014 & 22.9 \\
& sextet \#2 & 45.24 & 0.33 & 0.013 & 14.4 \\
& sextet \#3 & 42.17 & 0.34 & 0.010 & 15.4 \\
& sextet \#4 & 37.33 & 0.23 & 0.010 & 39.3 \\
& singlet & - & 0.21 & - & 8.0
\end{tabular}

The hysteresis loops measured for non-coated and silica-coated magnetite nanoparticles are presented in Fig. 4. For both samples non-zero coercive field and remanence values were measured. Their similar shapes indicate ferrimagnetic ordering originating from predominant volume fraction of large ferrimagnetic nanoparticles. The magnetization value measured at $\approx 500 \mathrm{kA} / \mathrm{m}$ for both samples is lower than this determined for bulk magnetite particles [15], therefore a presence of superparamagnetic component of the synthesized $\mathrm{Fe}_{3} \mathrm{O}_{4}$ cannot be excluded. 


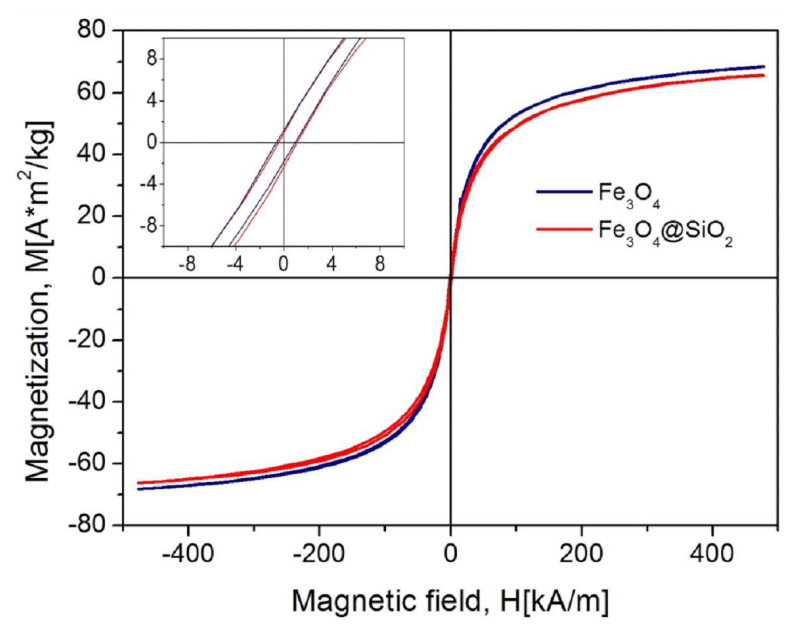

Fig. 4. Magnetic hysteresis loops measured for noncoated and silica-coated $\mathrm{Fe}_{3} \mathrm{O}_{4}$ nanoparticles.

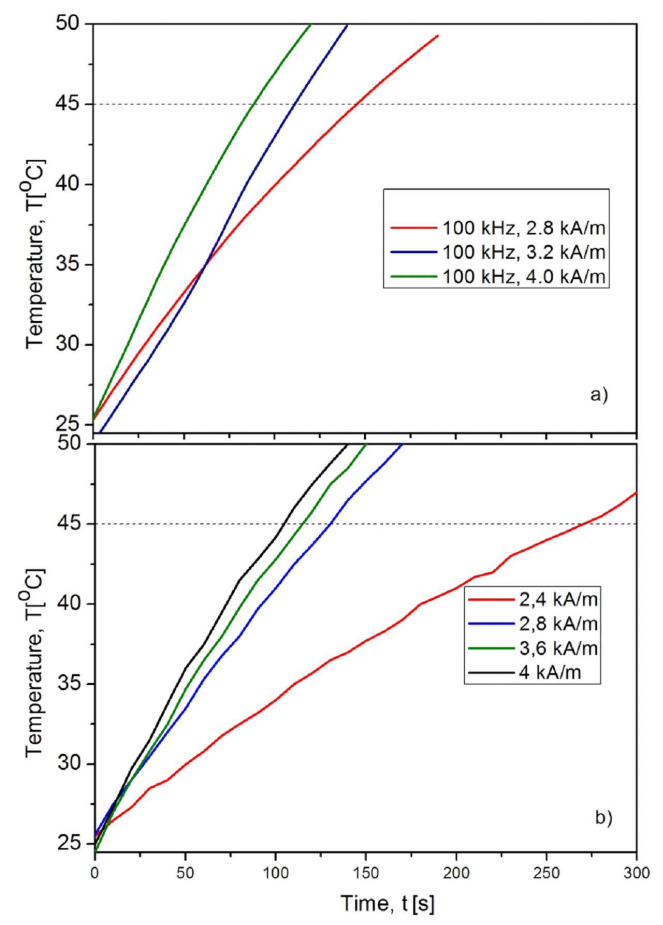

Fig. 5. Heating rate for 2 wt.\% (a) and 5 wt. \% (b) dispersion of $\mathrm{Fe}_{3} \mathrm{O}_{4}$ nanoparticles in glycerol for given amplitude of alternating external magnetic field.

The measurements of heating rate were performed on magnetite particles dispersed in glycerol for two different contents of the nanoparticles in the solution (2 wt\% and $5 \mathrm{wt} \%$ ). The results of measurements are presented in Fig. 5. The heating efficiency increases systematically with the increase of the amplitude of magnetic field. The specific loss power SLP was calculated using the formula [17, 18]:

$$
S L P=\frac{C}{m_{\mathrm{Fe}_{3} \mathrm{O}_{4}}} \frac{\mathrm{d} T}{\mathrm{~d} t},
$$

where $m_{\mathrm{Fe}_{3} \mathrm{O}_{4}}$ - mass of the magnetite nanoparticles, $T$ - temperature, $t$ - time, $C$ - heat capacity of the solution, calculated as

$$
C=\sum_{i=1}^{n} c_{i} m_{i},
$$

where $c_{i}$ - specific heat capacities for glycerol $(2.434 \mathrm{~J} /(\mathrm{g} \mathrm{K}))$ and magnetite $(0.62 \mathrm{~J} /(\mathrm{g} \mathrm{K}))$, and $m_{i}-$ mass of glycerol and magnetite nanoparticles, respectively. SLP parameters calculated for the samples are presented in Table III. Calculated heating rates as well

TABLE III

Mean heating rates $\mathrm{d} T / \mathrm{d} t$ and $S L P$ values of $\mathrm{Fe}_{3} \mathrm{O}_{4}$ dispersions (2 wt.\% and 5 wt.\%) in glycerol in AC magnetic fields of $100 \mathrm{kHz}$.

\begin{tabular}{c|c|c|c}
\hline & $H[\mathrm{kA} / \mathrm{m}]$ & $\frac{\mathrm{d} T}{\mathrm{~d} t}[\mathrm{~K} / \mathrm{min}]$ & $S L P[\mathrm{~W} / \mathrm{g}]$ \\
\hline \multirow{3}{*}{ wt.\% } & 2.8 & 5.5 & 11.0 \\
& 3.2 & 10.1 & 20.3 \\
& 4.0 & 10.3 & 20.6 \\
5 wt.\% & 2.4 & 4.8 & 4.3 \\
& 2.8 & 10.0 & 9.4 \\
& 3.6 & 12.0 & 10.8 \\
& 4.0 & 12.6 & 11.3
\end{tabular}

as SLP values for $2 \mathrm{wt} \%$ dispersion of magnetite nanoparticles are somewhat larger than those for the $5 \mathrm{wt} \%$ sample. This can be related to higher aggregation of $\mathrm{Fe}_{3} \mathrm{O}_{4}$ nanoparticles in $5 \mathrm{wt} \%$ dispersion. It is rather difficult to compare both heating rate and SLP with those reported by other authors, as it depends on the frequency and amplitude of external magnetic field. However, the SLP values are similar to those for maghemite nanoparticles measured at similar conditions [12]. To a certain extent these results are also consistent with SLP values measured for iron-oxide nanoparticles of similar diameter reported in [19], though they were carried out at different conditions.

\section{Conclusions}

Current studies were focused on determining the structural parameters of iron-oxide nanoparticles using the $\mathrm{X}$ ray diffractometry and the Mössbauer spectroscopy. It was shown that the co-precipitation process allows to obtain the single phase magnetite nanoparticles. To determine the unit cell parameters of the crystalline phase, the Rietveld refinement method was used. It was shown that the calculated lattice constant for both non-coated and silica-coated magnetite nanoparticles is higher than the starting value used in the calculations. This can be attributed to the disordered surface of nanoparticles and divergence from the stoichiometry as well as to the distribution of the nanoparticle sizes formed during coprecipitation. The full powder patter analysis has shown that magnetite non-coated and silica-coated particles had 
the average crystallite sizes of the order of $15 \mathrm{~nm}$. The Mössbauer studies have revealed differences in hyperfine structure of non-coated and $\mathrm{SiO}_{2}$-coated iron-oxide nanoparticles. This can be attributed to the interactions of the surface Fe ions of $\mathrm{Fe}_{3} \mathrm{O}_{4}$ nanoparticles with the silica-coating. Furthermore, the Mössbauer spectra analysis and measurements of hysteresis loops indicated a presence of minor fraction of the superparamagnetic component of the synthesized nanoparticles. The time dependences of temperature measured for the glycerol solutions of the magnetite nanoparticles have shown their strong dependences on the amplitude of external magnetic field.

\section{References}

[1] B.N. Ames, L. Swirsky Gold, $M u$ tat. Res./Fundament. Mol. Mech. Mutagen. 447, 3 (2000).

[2] V.T. DeVita Jr., S. Hellman, S.A. Rosenberg, Cancer Principles and Practice of Oncology, 7th ed., Lippincott Williams \& Wilkins, Philadelphia 2005.

[3] F. Roila, P.J. Hesketh, J. Herrstedt, Ann. Oncol. 17, 20 (2006).

[4] A. Skumiel, M. Izydorzak, M. Leonowicz, A.D. Pomogailo, G.I. Dzhardimalieva, Int. J. Thermophys. 32, 1973 (2011).

[5] M. Izydorzak-Wozniak, M. Leonowicz, J. Nanosci. Nanotechnol. 14, 2258 (2014).

[6] H. Chiriac, T. Petreus, E. Carasevici, L. Labusca, D. Herea, C. Danceanu, N. Lupu, J. Magn. Magn. Mater. 380, 13 (2015).
[7] S. Dutz, R. Hergt, Nanotechnology 25, 452001 (2014).

[8] A. Brezovich, in: Med. Phys. Monograph, American Institute of Physics, New York 1988, p. 82.

[9] Q.A. Pankhurst, J. Connolly, S.K. Jones, J. Dobson, J. Phys. D Appl. Phys. 36, 167 (2003).

[10] Y.H. Deng, D.W. Qi, C.H. Deng, X.M. Zhang, D.Y. Zhao, J. Am. Chem. Soc. 130, 28 (2008).

[11] M.A. Ghasemzadeh, Acta Chim. Slov. 62, 977 (2015).

[12] I. Smolkova, N. Kazantseva, V. Babayan, P. Smolka, H. Parmar, J. Vilcakova, O. Schneeweiss, N. Pizurova, J. Magn. Magn. Mater. 374, 508 (2015).

[13] I.S. Smolkova, N.E. Kazantseva, H. Parmar, V. Babayan, P. Smolka, P. Saha, Mater. Chem. Phys. 155, 178 (2015).

[14] A. Demir, A. Baykal, H. Sozeri, R. Topkaya, Synth. Met. 187, 75 (2014).

[15] A. Mitra, J. Mohapatra, S.S. Meena, C.V. Tomy, M. Aslam, J. Phys. Chem. C 118, 19356 (2014).

[16] B. Kalska-Szostko, M. Zubowska, D. Satuła, Acta Phys. Pol. A 109, 365 (2006).

[17] A. Jordan, P. Wust, H. Fahling, W. John, A. Hinz, R. Felix, Int. J. Hyperther. 25, 499 (2009).

[18] S. Laurent, S. Dutz, U.O. Hafeli, M. Mahmoudi, Adv. Coll. Interf. 166, 8e23 (2011).

[19] A. Figuerola, R. Di Corato, L. Manna, T. Pellegrino, Pharmacol. Res. 62, 126 (2010). 